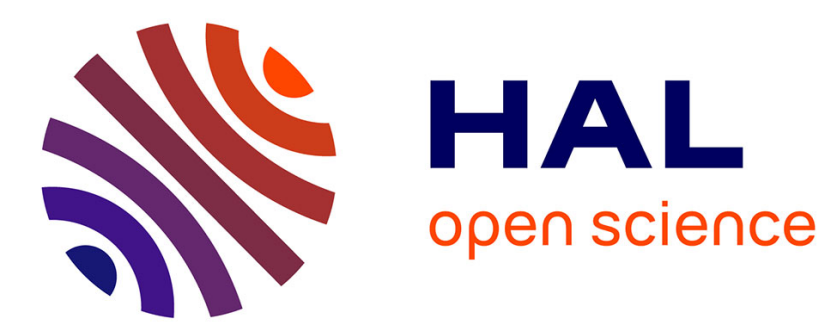

\title{
Unveiling the Role of Hot Charge-Transfer States in Molecular Aggregates via Nonadiabatic Dynamics
}

Daniele Fazzi, Mario Barbatti, Walter Thiel

\section{To cite this version:}

Daniele Fazzi, Mario Barbatti, Walter Thiel. Unveiling the Role of Hot Charge-Transfer States in Molecular Aggregates via Nonadiabatic Dynamics. Journal of the American Chemical Society, 2016, 138 (13), pp.4502-4511. 10.1021/jacs.5b13210 . hal-02288606

\section{HAL Id: hal-02288606 https://hal-amu.archives-ouvertes.fr/hal-02288606}

Submitted on 15 Sep 2019

HAL is a multi-disciplinary open access archive for the deposit and dissemination of scientific research documents, whether they are published or not. The documents may come from teaching and research institutions in France or abroad, or from public or private research centers.
L'archive ouverte pluridisciplinaire HAL, est destinée au dépôt et à la diffusion de documents scientifiques de niveau recherche, publiés ou non, émanant des établissements d'enseignement et de recherche français ou étrangers, des laboratoires publics ou privés. 


\title{
Unveiling the role of hot charge-transfer states in molecular aggregates via nonadiabatic dynamics
}

\author{
Daniele Fazzi ${ }^{1}$, Mario Barbatti ${ }^{2}$, Walter Thiel ${ }^{1}$ \\ ${ }^{1}$ Max-Planck-Institut für Kohlenforschung, \\ Kaiser-Wilhelm-Platz 1, D-45470 Mülheim an der Ruhr, Germany \\ ${ }^{2}$ Aix Marseille Université, CNRS, ICR UMR7273, 13397 Marseille, France.
}

\begin{abstract}
Exciton dynamics governs energy transfer and charge generation in organic functional materials. We investigate high-energy nonadiabatic excited-state dynamics for a bi-thiophene dimer to describe time-dependent excitonic effects in molecular aggregates. We show that the lowest excited states are populated on the sub-picosecond time scale. These states are localized and unproductive in terms of charge separation. Productive high-energy charge-transfer (CT) states are populated within 50 fs during exciton deactivation, but they are short-lived ( $100 \mathrm{fs})$ and quickly transfer their population to lower states. Our simulations offer molecular-level insights into ultrafast photoinduced charge separation potentially triggered by hot CT states in solid-state organic materials. Design rules are suggested to increase hot exciton lifetimes, favouring the population of CT states as gateways for direct charge generation. These rules may boost the CT quantum yield by depleting unproductive recombination channels.
\end{abstract}




\section{INTRODUCTION}

Excitons are the primary outcome of light-matter interactions. Their formation and relaxation play a key role in a variety of areas, including chemical reaction dynamics ${ }^{1}$, ultrafast electron-nuclei processes in organic and inorganic materials ${ }^{2-4}$, dynamics of semiconductors and quantum $\operatorname{dots}^{5}$, and light harvesting and energy transfer in photosynthetic systems ${ }^{6}$. In particular, ultrafast exciton dynamics govern the energy flow and the charge generation/recombination mechanisms in organic $\pi$-electron conjugated materials and interfaces ${ }^{7}$.

In the last two decades, our understanding of ultrafast phenomena ruling exciton dynamics and charge generation processes in organic materials has significantly improved. This progress took place in the wake of scientific and industrial developments in photovoltaics, electronics, spintronics, and thermoelectrics, with strong focus on soft materials ${ }^{8-15}$.

However, several features of exciton and charge generation dynamics at the molecular scale are still obscure. The usage of fairly similar concepts such as frontier orbitals and interfacial states is still common in the literature. Even the role of one of the primary process, the high-energy (hot) exciton relaxation, is still under debate ${ }^{16-20}$ in the organic and hybrid photovoltaics communities: it is unclear whether hot excitons would favour an instantaneous electron-hole charge separation, thus increasing the overall charge generation efficiency, or alternatively lead to unproductive nonradiative decay toward low-lying electron-hole bound states.

Experimental evidence of fast ( $\sim 30-100 \mathrm{fs})$ electron transfer processes and polaron formation from photoinduced hot excitons has recently been reported for thiophene-based molecules ${ }^{21}$, acenes ${ }^{22}$, diverse polymers $^{23}$, and polymer/fullerene interfaces ${ }^{17,18,21,24-26}$. This ultrafast population of hot CT states competes with nonradiative mechanisms within the manifold of excited states ${ }^{27}$. Internal relaxation can bring hot excitons to the bottom of the excited-state band, thus reducing the lifetime of hot CTs and allowing for nonradiative pathways and recombination processes that are detrimental for efficient charge generation ${ }^{28}$. 
An appropriate theoretical description and modelling of ultrafast exciton relaxation in organic aggregates is urgently needed ${ }^{29,30}$. Pioneering recent studies by Rossky et al. ${ }^{31,32}$, Tamura et al. ${ }^{33,34}$, Tretiak et al. ${ }^{4,13,35}$, and Prezhdo et al. ${ }^{10,36,37}$ describe the nonadiabatic excited-state dynamics of mediumsized systems based on different approximations. Limited to model Hamiltonians, single-determinant Kohn-Sham treatments or low-dimensional 1-D systems, these simulations have contributed to the understanding of the dynamic processes, but without addressing in detail the nature of the electronic states accessed during the relaxation process. This knowledge gap is tackled in our present work.

To understand ultrafast exciton relaxation phenomena and the population of hot CT states from an atomistic and time-dependent viewpoint, we have performed full-dimensional nonadiabatic excitedstate dynamics simulations based on linear-response time-dependent density functional theory $(\text { TDDFT) })^{29,38}$ for the bi-thiophene stacking dimer (Figure 1), a molecular aggregate representative of a large class of solid-state organic $\pi$-conjugated materials. Our aim is to understand how hot excitons relax and whether we can control their lifetime. For this purpose, we use nonadiabatic excited-state dynamics to understand and predict $i$ ) the excited-state relaxation time scales for high-energy excitations; ii) the interplay between inter- and intra-molecular nonadiabatic relaxations; iii) the population of CT states; (iv) exciton delocalization/localization processes; and v) the nuclear vibrations governing exciton dynamics in thiophene-based molecular aggregates.

Our working hypothesis is that productive charge separation and transfer processes in organic electronics and photovoltaics depend on the population and lifetime of CT states. However, such CT states are usually not among the lowest states in the manifold of excited states in organic homo- and hetero-junctions ${ }^{39}$. If the lifetime of high-lying (hot) excited states is extended, the time the dimer spends in CT states rises, which increases the odds of productive charge separation. In this spirit, we propose a new goal for the design of functionalized molecular aggregates: if we can modify the excitedstate electronic structure by introducing energy gaps between high- and low-energy bands, this will disfavour unproductive ultrafast exciton relaxation and increase the residence time of the photoexcitation on productive hot CTs (see Scheme 1). The main assumption underlying this hypothetical scenario is that charge separation is triggered by a charge transfer process between two 
monomers. However, it might also be possible that charge transfer states extended over several monomers act as triggers for (long-range) charge separation ${ }^{40}$. We do not address this possibility here, but only point out that in principle it can be studied using the same methodology.
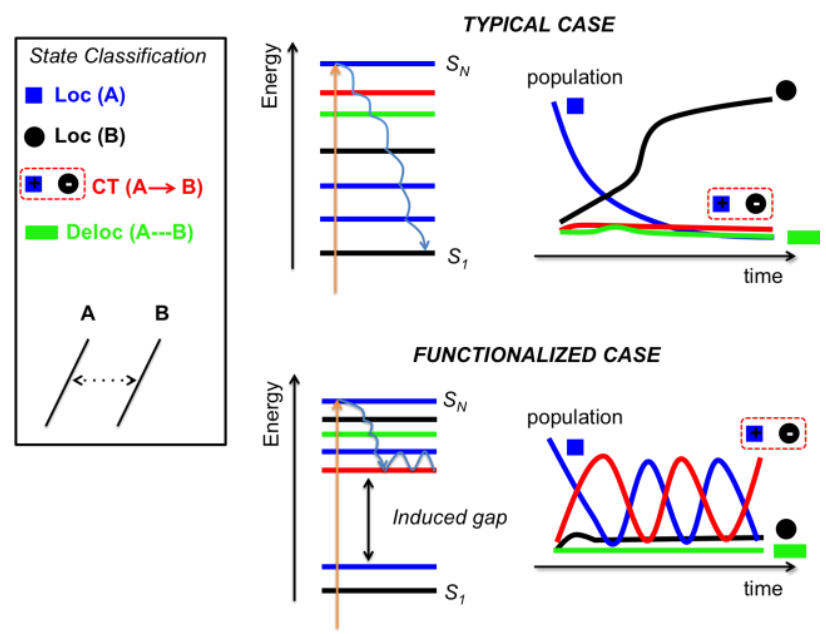

Scheme 1. Left side: colour code for excited-state classification and sketch of a molecular dimer. Right side: excited-state classification and time-dependent state population of a typical (top) and a functionalized (bottom) molecular aggregate.

\section{COMPUTATIONAL METHODS}

Ground- and excited-state properties of the bi-thiophene dimer were investigated with DFT and TDDFT. The ground-state and the first three excited-state equilibrium geometries were optimized at the $\omega \mathrm{B} 97 \mathrm{XD}$ level, using the $6-31 \mathrm{G}^{*}, 6-311+\mathrm{G}^{*}$, and aug-ccpVTZ basis sets. This approach, which incorporates long-range and dispersion corrections, has been previously shown to provide semiquantitatively correct predictions for this type of systems ${ }^{39}$. Static calculations were done both in vacuum and in a dielectric using the conductor polarisable continuum model (CPCM). Ground-state geometries and vertical electronic transitions were also evaluated using the algebraic diagrammatic construction to second order (ADC(2)) ${ }^{41}$, combined with SVP and def2-TZVP basis sets. Further details are given in the Supplementary Information (Supplementary Note 1) including a more thorough description of the computational procedures and additional numerical results. 
Absorption cross sections were computed with the nuclear ensemble method based on harmonicoscillator Wigner distributions. These calculations employed the $\omega \mathrm{B} 97 \mathrm{XD}$ functional and the $6-31 \mathrm{G}^{*}$ and $6-311+\mathrm{G}^{*}$ basis sets, both in vacuum and in a dielectric continuum (see Supplementary Data). 150 points were considered for spectrum generation.

Nonadiabatic excited-state dynamics were done with TDDFT using the fewest-switching surface hopping (FSSH) method as implemented in NEWTON-X interfaced with Gaussian 0942. Dynamics were run at the TD- $\omega \mathrm{B} 97 \mathrm{XD} / 6-31 \mathrm{G}^{*}$ level, using a microcanonical ensemble. The time step for integration of the classical equations was $0.5 \mathrm{fs}$. The integration of the quantum equations was done with 0.025 fs steps using interpolated properties between classical steps. Time-dependent coefficients were corrected for decoherence effects as described in ref. ${ }^{43}$.

The large density of states in the dimers leads to numerous state crossings. In particular, the occurrence of trivial crossings between non-interacting or weakly interacting states has been recognized as potentially challenging for FSSH. In such cases, conventional FSSH becomes costly, requiring very small time steps ${ }^{44,45}$. This problem has been tackled by different strategies to track down the diabatic nature of the crossings, including the local diabatization method ${ }^{46,47}$, the self-consistent $\mathrm{FSSH}^{45}$, the flexible surface hopping techninque ${ }^{48}$, the global flux surface hopping ${ }^{49}$, and the Min Cost algorithm ${ }^{44}$. In the present work, nonadiabatic couplings between excited states were computed from time-dependent wavefunction overlap integrals as proposed by Hammes-Schiffer and Tully ${ }^{50}$ based on wavefunctions built through Casida's Ansatz ${ }^{51}$. This approach has been extensively used for computating nonadiabatic couplings in dynamics simulations ${ }^{52-55}$ and has been found to be very stable even in the presence of trivial crossings ${ }^{47}$.

Due to the limitations of the linear-response TDDFT and Kohn-Sham DFT methods in providing reliable electronic states near intersections with the ground state, nonadiabatic transitions between the first excited state and the ground state were not computed. Trajectories were run for a maximum of 300 fs or until a crossing with the ground state (within $0.15 \mathrm{eV}$ ) was reached. In such cases, the last time step was taken to indicate internal conversion to the ground state. Under this assumption, the occupation 
of the ground state and the correction to the $S_{1}$ occupation were computed at each time step as reported in ref. ${ }^{56}$.

The excited states were classified as localized, delocalized, or charge-transfer using the method introduced and discussed in refs. ${ }^{39,57}$. In brief, the molecular aggregate is split in two units $A$ and $B$ (in this case, the two monomers); then the contribution of each molecular orbital to each unit is computed with a Mulliken partition. With this information, the amount of localization $\left(\Sigma P_{A}^{I}\right)$ and of charge transfer $\left(\Delta P_{A}^{I}\right)$ for each electronic state $I$ is computed for unit $A$ based on a configuration-interaction wavefunction approximation, using linear-response coefficients. Finally, the densities $\Sigma P_{A}^{I}$ and $\Delta P_{A}^{I}$ are compared to predefined thresholds to classify the states.

\section{RESULTS AND DISCUSSION}

\section{Absorption spectrum}

The computed TDDFT vertical excitations and absorption cross sections for the bi-thiophene monomer and the van-der-Waals (vdW) dimer are reported in Figure 1.

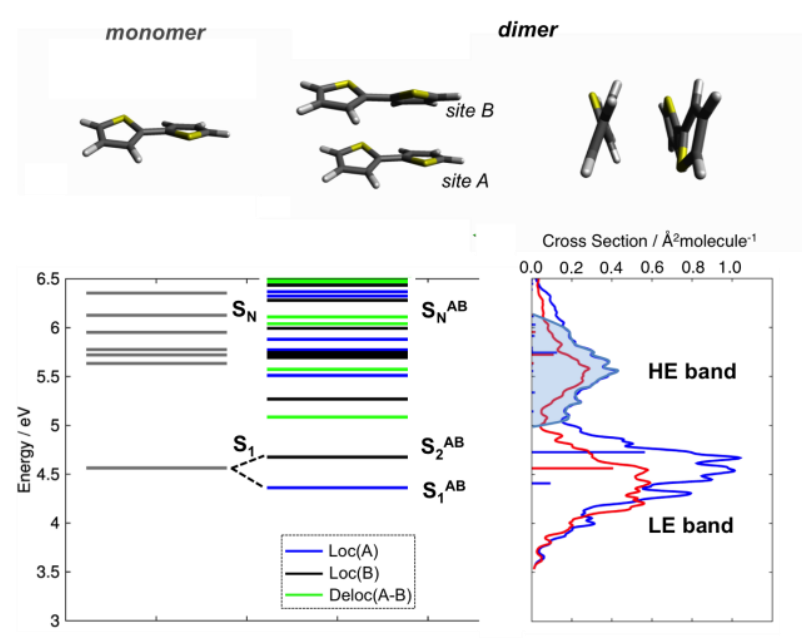

Figure 1. Top: DFT $\left(\omega \mathrm{B} 97 \mathrm{XD} / 6-31 \mathrm{G}^{*}\right)$ molecular structures for the bi-thiophene monomer and dimer (lateral and side views). Bottom left: TDDFT ( $\omega$ B97XD/6-31G*) vertical transition energies for the bi-thiophene monomer and dimer, and excited-state classification. Bottom right: absorption cross 
sections of monomer (red) and dimer (blue), and oscillator strengths (horizontal bars); the photoexcited high-energy (HE) band is represented by the shaded area.

Just like in other noncovalent organic dimers, ${ }^{58}$ the ground-state structure of bi-thiophene dimer is asymmetric causing a split of the low-lying excited states $\left(\mathrm{S}_{1}{ }^{\mathrm{AB}}, \mathrm{S}_{2}{ }^{\mathrm{AB}}\right)$. Analysis of the dimer excited states shows $\mathrm{S}_{1}{ }^{\mathrm{AB}}$ and $\mathrm{S}_{2}{ }^{\mathrm{AB}}$ to be localized within each monomer. Delocalized excited states are predicted at higher energies, and excited states with $\mathrm{CT}$ character are not present below $6.5 \mathrm{eV}$ in the vertical spectrum. The dimer absorption cross section gives rise to two bands, one at low energy (LE, $\sim 4.5 \mathrm{eV})$ and another at high energy $(\mathrm{HE}, \sim 5-6 \mathrm{eV})$. A manifold of excited states contributes to the HE band, and among them $\mathrm{S}_{8}$ has non-negligible oscillator strength $(f=0.13)$. At distorted ensemble geometries, the bright state may also occur as $\mathrm{S}_{9}$.

\section{High-energy nonadiabatic dynamics}

TDDFT nonadiabatic excited-state dynamics was initiated in the HE band, restricting the excitation to the $5.5 \pm 0.5 \mathrm{eV}$ spectral window (shaded area in Figure 1). A total of 30 trajectories, considering 10 coupled singlet electronic states in each case, were initiated in the $\mathrm{S}_{9}$ state and propagated up to $300 \mathrm{fs}$ or until the $\mathrm{S}_{1}{ }^{\mathrm{AB}} / \mathrm{S}_{0}{ }^{\mathrm{AB}}$ gap became smaller than $0.15 \mathrm{eV}$. Six trajectories reached the $\mathrm{S}_{1}{ }^{\mathrm{AB}} / \mathrm{S}_{0}{ }^{\mathrm{AB}}$ crossing during the simulations, while 24 had still large $\mathrm{S}_{1}{ }^{\mathrm{AB}} / \mathrm{S}_{0}{ }^{\mathrm{AB}}$ energy gaps at $300 \mathrm{fs}$.

Figure 2 shows the occupations of the states in the HE band (sum over $\mathrm{S}_{3}{ }^{\mathrm{AB}}$ to $\mathrm{S}_{9}{ }^{\mathrm{AB}}$ ) and the $\mathrm{LE}$ band (sum over $\mathrm{S}_{1}{ }^{\mathrm{AB}}$ and $\mathrm{S}_{2}{ }^{\mathrm{AB}}$ ) and also of the $\mathrm{S}_{0}{ }^{\mathrm{AB}}$ ground state as a function of time. The HE band starts to transfer population to LE after 15 fs. Its population decays exponentially with a time constant of $122 \mathrm{fs}$. The LE band transfers about $20 \%$ of the population to the ground state, with a time constant of $102 \mathrm{fs}$ (see Supplementary Note 2).

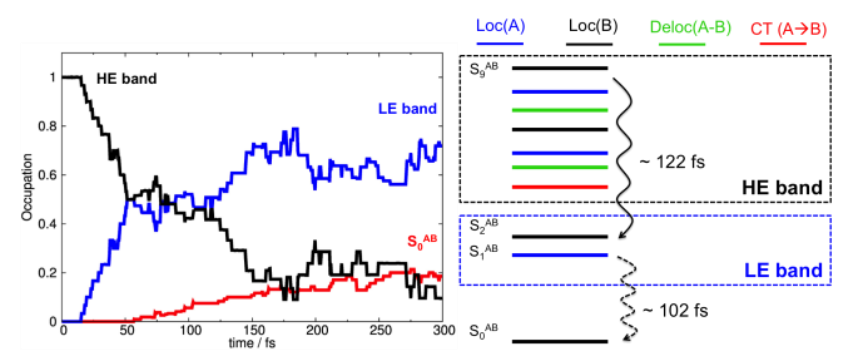


Figure 2. TDDFT $\left(\omega \mathrm{B} 97 \mathrm{XD} / 6-31 \mathrm{G}^{*}\right)$ nonadiabatic excited-state dynamics initiated by populating a high-energy excited state $\left(\mathrm{S}_{9}{ }^{\mathrm{AB}}\right)$. Left: state occupations $v$ s. time. Right: sketch of ultrafast relaxation within the manifold of high-energy excited states (HE band) toward the low-energy states (LE band, $\mathrm{S}_{1}{ }^{\mathrm{AB}}$ and $\mathrm{S}_{2}{ }^{\mathrm{AB}}$ ) and of nonradiative relaxation to the ground state.

These findings can be compared to very recent $\mathrm{TDDFT}^{56}$ and $\mathrm{ADC}(2)^{59}$ nonadiabatic excited-state dynamics performed on an isolated bi-thiophene monomer. In this case, HE-band excitation leads to over $80 \%$ occupation of $S_{0}$ within $300 \mathrm{fs}$, while for the dimer the fraction is only $19 \%$. Therefore, the ultrafast nonradiative decay through $\mathrm{S}_{1} / \mathrm{S}_{0}$ crossing is significantly delayed in the $\mathrm{vdW}$ dimer compared to the monomer.

To better understand the exciton dynamics and the transfer processes in the dimer, two exemplary trajectories are illustrated in Figure 3. Traj A is representative of those remaining in the excited-state manifold during the entire simulation, while traj $B$ is an example of those few that reach the $S_{1} / S_{0}$ crossing before $300 \mathrm{fs}$.

In traj $\mathrm{A}$, the photoexcited dimer decays from a high-energy excited state $\left(\mathrm{S}_{9}{ }^{\mathrm{AB}}\right)$ to the low-lying $\mathrm{S}_{2}{ }^{\mathrm{AB}}$ and $\mathrm{S}_{1}{ }^{\mathrm{AB}}$ states in $150 \mathrm{fs}$. Thereafter, the dynamics continues in the LE regime and does not reach the ground state. In traj $\mathrm{B}$, the photoexcited system relaxes from $\mathrm{S}_{9}{ }^{\mathrm{AB}}$ to $\mathrm{S}_{1}{ }^{\mathrm{AB}}$ in $\sim 30 \mathrm{fs}$ and arrives at the $\mathrm{S}_{1}{ }^{\mathrm{AB}} / \mathrm{S}_{0}{ }^{\mathrm{AB}}$ intersection seam in about $100 \mathrm{fs}$.

To gain insight into the different deactivation mechanisms and character of the excited states involved in the dynamics, we have monitored in each time step the electronic-density difference between the occupied excited state and the ground state for these two trajectories. This information is also plotted in Figure 3. Three types of electronic states are observed during the dynamics: localized, delocalized, and CT states.

In traj A, a localized state is initially populated (Figure 3). Within $\sim 50 \mathrm{fs,} \mathrm{this} \mathrm{high-energy} \mathrm{exciton}$ evolves into a hot $\mathrm{CT}$ state, which features a net electron-hole separation between the two monomers. This CT state persists up to $150 \mathrm{fs}$, remaining populated for $100 \mathrm{fs}$. After $150 \mathrm{fs}$, the low-lying states are populated and energy transfer occurs between the two bi-thiophene units, leading to an oscillation between localized and delocalized states, with no further sign of a CT state. 
In traj $\mathrm{B}$, the dynamics starts in a delocalized state. The inter-molecular exciton localizes within $30 \mathrm{fs}$ (Figure 3) and remains localized until the crossing to the ground state takes place. The radiationless deactivation mechanism to the ground state resembles that occurring in the bi-thiophene monomer: a ring-opening process. ${ }^{56}$

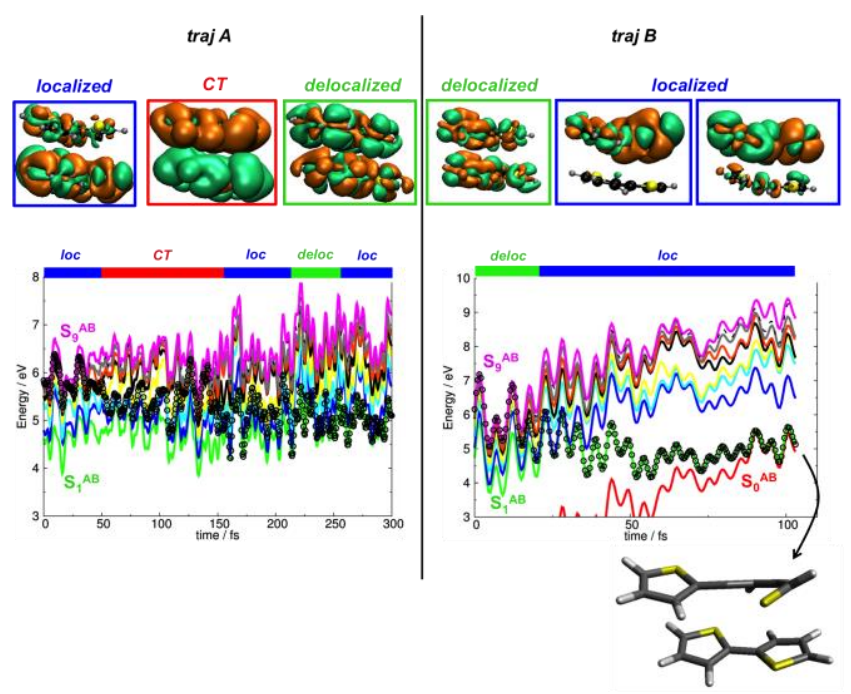

Figure 3. Two exemplary trajectories: left side $-\operatorname{traj} \mathrm{A}, t=0-300 \mathrm{fs}$; right side $-\operatorname{traj} \mathrm{B}, t=0-$ $100 \mathrm{fs}$. Top: snapshots of the electronic-density difference between the occupied excited state and the ground state for typical localized (loc), delocalized (deloc), or CT states; orange/green regions indicate loss/gain of electron density upon excitation. Bottom: time evolution of the excited-state energies (TD$\left.\omega \mathrm{B} 97 \mathrm{XD} / 6-31 \mathrm{G}^{*}\right)$ with indication of the prevailing state character. Also shown is the molecular structure close to the $\mathrm{S}_{1}{ }^{\mathrm{AB}} / \mathrm{S}_{0}{ }^{\mathrm{AB}}$ intersection seam featuring the typical thiophene ring opening.

\section{Role of the ultrafast nuclear oscillations in the hot exciton dynamics}

The time-dependent evolution of the excited states is governed by ultrafast nuclear oscillations, namely: i) CS vibrations (Figure 4), ii) ring puckerings, and iii) dihedral rotations $\angle$ (SCCS). The time scales for processes $i$ and $i i$ are shorter than for iii (50-100 fs vs. 250-300 fs), making CS bond elongations and ring puckerings the major nuclear motions contributing to nonadiabatic relaxation. Here we discuss the role of CS vibrations, being the most effective deactivation channel, while ring puckerings and dihedral rotations are addressed in Supplementary Note 3.

For traj A, in the early stages of the relaxation process ( $t \leq 150 \mathrm{fs})$, the CS stretchings control the ultrafast nonadiabatic transitions amongst high-lying excited states, bringing the dimer from the initial 
state $\left(\mathrm{S}_{9}{ }^{\mathrm{AB}}\right)$ to the hot $\mathrm{CT}$ state. Once the population is transferred from the hot $\mathrm{CT}$ to the $\mathrm{LE}$ band $\left(\mathrm{S}_{3}{ }^{\mathrm{AB}}\right.$ $\rightarrow \mathrm{S}_{2}{ }^{\mathrm{AB}}, t \sim 150 \mathrm{fs}$ ), exciton localization (intra-molecular) and delocalization (inter-molecular) processes occur (Figure 4). In the LE band, exciton localization tends to take place whenever the amplitude of a CS vibration is larger (smaller) than a quantity we call the average Bond Length Amplitude Oscillation $(\langle B L A O\rangle$, grey horizontal lines in Figure 4). It is defined as: $\langle B L A O\rangle=\langle B L\rangle \pm \Delta$, where $\langle B L\rangle$ is the average $\mathrm{CS}$ bond length, as computed at the equilibrium ground-state geometry of the dimer $\left(\mathrm{S}_{0}{ }^{\mathrm{AB}}\right.$ state), and $\Delta$ is the average CS bond length fluctuation around $\langle B L\rangle$, as extracted from a $300 \mathrm{fs} S_{1}$ excited-state dynamics run of a single bi-thiophene molecule ${ }^{56} .\langle B L A O\rangle$ can be interpreted as an oscillation interval that allows us to decide whether exciton localization occurs (CS vibrations beyond $\langle B L A O\rangle$ interval) or not (CS vibrations within $\langle B L A O\rangle$ interval), once the LE band is populated. At the $\omega \mathrm{B} 97 \mathrm{XD} / 6-31 \mathrm{G}^{*}$ level, $\langle B L\rangle=1.73 \AA$ and $\Delta= \pm 0.1 \AA$.

In traj $\mathrm{A}$, following the population of the LE band ( 150 fs), exciton localization occurs in two time windows, $150 \leq t_{1} \leq 200 \mathrm{fs}$ and $t_{2} \geq 250 \mathrm{fs}$ (Figure 4-left), when the amplitude of some CS vibrations is larger than $\langle B L A O\rangle$. For traj $\mathrm{B}$, the CS bonds tend to oscillate between 1.6 and $2.0 \AA$ (similar to traj A); however, at around $30 \mathrm{fs}$ one CS bond on molecular site B starts to strongly stretch (> $2 \AA$ ), soon exceeding the upper limit of $\langle B L A O\rangle$ and continuing until it cleaves (Figure 4-right). This process leads to exciton localization and subsequent radiationless deactivation through the $\mathrm{S}_{1}{ }^{\mathrm{AB}} / \mathrm{S}_{0}{ }^{\mathrm{AB}}$ crossing (Figure 3). The mechanism is similar to the $\mathrm{S}_{1} \rightarrow \mathrm{S}_{0}$ nonradiative ring-opening pathway found for the isolated bi-thiophene monomer in Ref ${ }^{56}$.
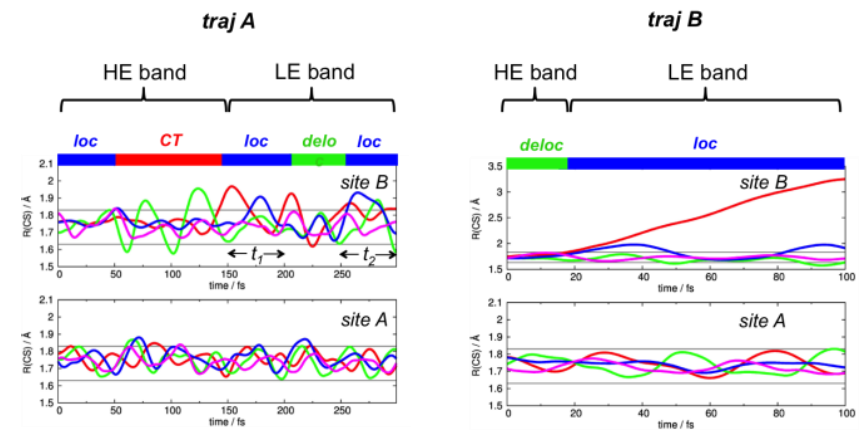

Figure 4. Variation of the CS distances in the bi-thiophene vdW dimer (molecular sites A and B) and classification of the occupied excited states during the nonadiabatic dynamics. Left: $\operatorname{traj} \mathrm{A}(t=0-$ $300 \mathrm{fs})$; right: traj B $(t=0-100 \mathrm{fs})$. Grey lines: $\langle B L A O\rangle$ (see text). 
The analysis of all trajectories reaching the maximum simulation time (without returning to the ground state) shows the same exciton dynamics as observed for traj A: $i$ ) an initial decay ( 20-50 fs) from high-lying excitons to hot CT states; ii) survival and evolution of the hot CT states for $\sim 50-100$ fs; iii) transfer to the low-lying excited states $\left(\mathrm{S}_{2}{ }^{\mathrm{AB}}, \mathrm{S}_{1}{ }^{\mathrm{AB}}\right)$ originating from the symmetry breaking; and iv) oscillations between localized and delocalized states in the LE regime, governed by CS vibrations.

The ultrafast population of hot $\mathrm{CT}$ states, as computed from our TDDFT nonadiabatic dynamics, matches well with experimental studies on a variety of organic materials ${ }^{4,18,21-23,32}$, which report formation of hot CTs within 30-60 fs and further ultrafast photoinduced polaron generation.

We have already mentioned that the fraction of trajectories reaching the intersection with the ground state is much smaller in the dimer than in the monomer. The nonradiative pathways toward the ground state are controlled by CS bond stretchings, which destabilize the $\mathrm{S}_{0}$ state and stabilize $\mathrm{S}_{1}{ }^{56,60}$. Therefore, to understand the reason for the fewer radiationless processes in the dimer, we compare the excited-state potential energy profiles along the CS stretching and the average CS fluctuations in the monomer and in the dimer (Figure 5).

a) $\quad \mathrm{LE}$ band $\left(\mathrm{S}_{1}{ }^{\mathrm{AB}}, \mathrm{S}_{2}{ }^{\mathrm{AB}}\right) / \mathrm{S}_{0}{ }^{\mathrm{AB}}$

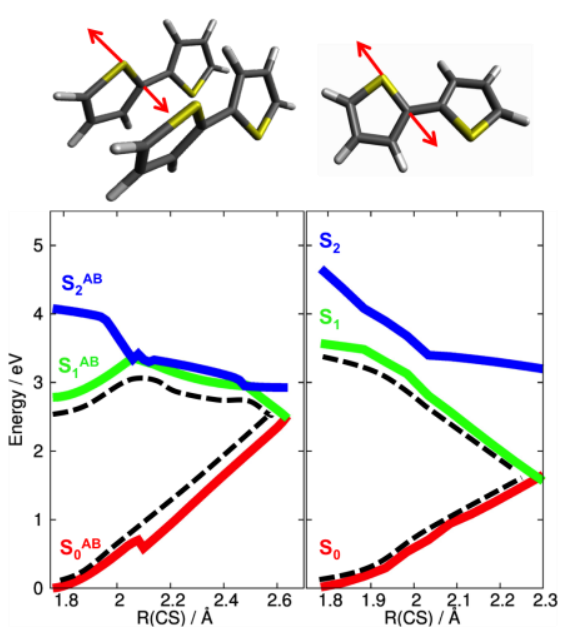

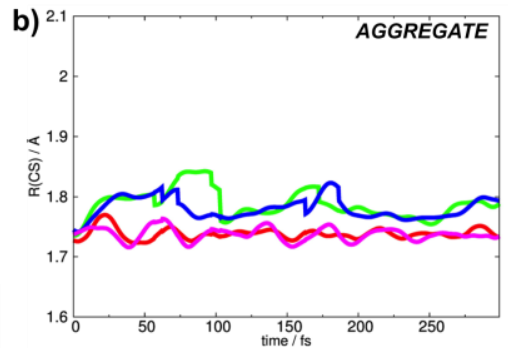

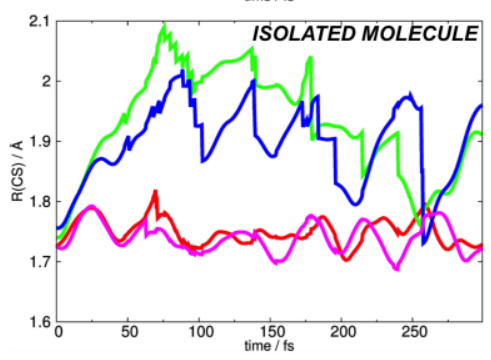

c) $\quad \mathrm{HE}$ band $\left(\mathrm{S}_{\mathrm{n}}{ }^{\mathrm{AB}}\right) / \mathrm{S}_{2}{ }^{\mathrm{AB}}$
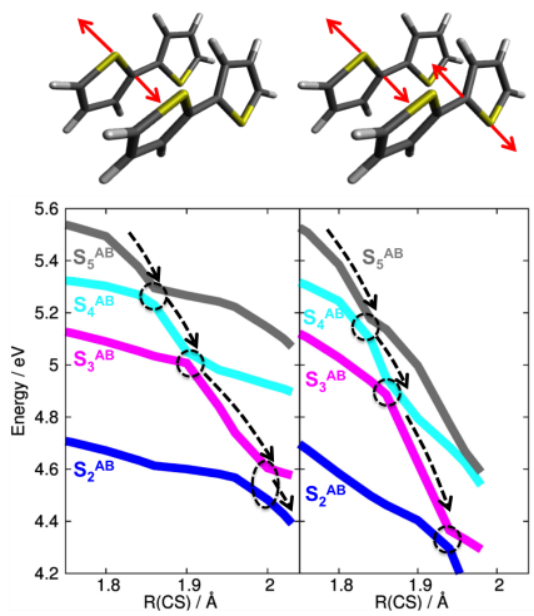

Figure 5. Panel a): TDDFT $\left(\omega \mathrm{B} 97 \mathrm{XD} / 6-31 \mathrm{G}^{*}\right)$ relaxed potential energy profiles of LE states $\left(\mathrm{S}_{2}{ }^{\mathrm{AB}}, \mathrm{S}_{1}{ }^{\mathrm{AB}}\right)$ and ground state $\left(\mathrm{S}_{0}{ }^{\mathrm{AB}}\right)$ for the bi-thiophene dimer (left side). The profiles are computed by optimizing the $\mathrm{S}_{1}{ }^{\mathrm{AB}}$ state as a function of the CS distance (in increments of $0.02 \AA$ ) starting from the $\mathrm{S}_{1}{ }^{\mathrm{AB}}$ equilibrium structure; analogous curves are shown for the monomer at the right; dashed lines are 
meant to guide the eye. Panel b): average CS distances for the bi-thiophene dimer (top) and monomer (bottom), as extracted from nonadiabatic excited-state dynamics; data for the monomer from Ref. ${ }^{56}$. Panel c): single-point TDDFT ( $\left.\omega \mathrm{B} 97 \mathrm{XD} / 6-31 \mathrm{G}^{*}\right)$ potential energy profiles of HE excited states $\left(\mathrm{S}_{3}{ }^{\mathrm{AB}}{ }_{-}\right.$ $\mathrm{S}_{5}{ }^{\mathrm{AB}}$ ) and $\mathrm{S}_{2}{ }^{\mathrm{AB}}$, as function of one CS distance in one bi-thiophene unit (left) or of two CS distances belonging to different molecular sites (right, dashed arrows to guide the eye).

As shown in Figure $5 \mathrm{a}$, the crossing between the $\mathrm{S}_{1}{ }^{\mathrm{AB}}$ state and the ground state is reached via an activated process in the dimer. From the $\mathrm{S}_{1}{ }^{\mathrm{AB}}$ minimum, a potential energy barrier of $\sim 1 \mathrm{eV}$ needs to be overcome to reach this crossing. In the case of the bi-thiophene monomer, the process is barrierless instead. The barrier in the $\mathrm{S}_{1}{ }^{\mathrm{AB}}$ profile of the dimer impedes radiationless transitions to the ground state, which explains the slower return to the $\mathrm{S}_{0}{ }^{\mathrm{AB}}$ state, compared to the monomer.

The source of this strong difference between the reaction paths in the monomer and the dimer along the CS stretching coordinate is due to the energetics of the lowest $\pi \pi^{*}$ state in the region near the $\mathrm{S}_{1}$ minimum $(\mathrm{R}(\mathrm{CS})<2 \AA)$. As expected, in the monomer, the CS stretching has little effect on the energetics of this state. In the dimer, however, the CS stretching strongly destabilizes the lowest $\pi \pi^{*}$ state because it breaks the intermolecular interactions that had stabilized the $\pi \pi^{*}$ in first place. As result, while in the monomer the path to the crossing to the $\pi \sigma^{*}$ state (which is strongly stabilized along this coordinate and gives rise to the intersection with $\mathrm{S}_{0}$ ) is almost barrierless, in the dimer a large barrier connects the $\pi \pi^{*}$ to the $\pi \sigma^{*}$ state. Thus, the $\pi-\pi$ stacking interactions change the shape of the excitedstate potential energy surfaces and thus ultimately govern the exciton dynamics, ${ }^{61}$ as also indirectly observed via Raman spectroscopy in the case of oligothiophene aggregates ${ }^{62}$. Additional information about the reaction path along the CS stretching is provided in the Supplementary Note 3 (Points 4 and $5)$.

As shown in Figure $5 \mathrm{~b}$, the mean CS bond length variations are smaller in the dimer $(1.7 \AA<$ $\mathrm{R}(\mathrm{CS})<1.85 \AA)$ than in the monomer $(1.7 \AA<\mathrm{R}(\mathrm{CS}) \leq 2.1 \AA)$, which lowers the chances for deactivation by the ring-opening mechanism. Thus, the $S_{1}{ }^{A B}$ potential energy barrier and the hindering of the CS oscillations in the dimer have the overall effect of increasing the lifetime of the low-energy 
excited states (e.g. $\mathrm{S}_{2}{ }^{\mathrm{AB}}$ and $\mathrm{S}_{1}{ }^{\mathrm{AB}}$ ) and thus lowering the odds of radiationless deactivation to the ground state.

The CS bond stretchings play another fundamental role even before the $\mathrm{S}_{1}{ }^{\mathrm{AB}}$ state is populated: they are ultimately responsible for driving the exciton relaxation through the HE band and on to the LE band. This is illustrated in the potential energy profiles from rigid scans shown in Figure 5c. These profiles were computed for two cases: $i$ ) elongation of one CS bond (Error! Reference source not found.c, left) from its equilibrium value $(\sim 1.72 \AA)$ and $i$ ) concomitant stretching of two inner CS bonds belonging to different units (Figure 5c, right). Both motions destabilize the HE states and lead to state crossings that may act as funnels for exciton relaxation through the HE band and for the population of $\mathrm{S}_{2}{ }^{\mathrm{AB}}$ in the LE band.

The stabilization of $\mathrm{S}_{3}{ }^{\mathrm{AB}}$ leading to a state crossing with $\mathrm{S}_{2}{ }^{\mathrm{AB}}$ is of special interest. As discussed before (traj A), the $\mathrm{S}_{3}{ }^{\mathrm{AB}} \rightarrow \mathrm{S}_{2}{ }^{\mathrm{AB}}$ relaxation represents the population transfer from a hot $\mathrm{CT}$ state, belonging to the HE band, to a localized state belonging to the LE band. The faster the $\mathrm{S}_{3}{ }^{\mathrm{AB}} \rightarrow \mathrm{S}_{2}{ }^{\mathrm{AB}}$ relaxation occurs, the shorter is the lifetime of hot $\mathrm{CT}$ states; and the lower is the probability of charge separation. As can be seen from Figure 5c, the stretching of the CS bonds governs the evolution of the $\mathrm{S}_{3}{ }^{\mathrm{AB}}{ }_{-} \mathrm{S}_{2}{ }^{\mathrm{AB}}$ energy gap $\Delta E\left(\mathrm{~S}_{3}{ }^{\mathrm{AB}}-\mathrm{S}_{2}{ }^{\mathrm{AB}}\right)$, and thus the deactivation from $\mathrm{HE}$ to LE bands. Although we cannot exclude that $\mathrm{S}_{2}$ may have CT character at some specific geometries, the data analysis shows that non-CT regions of the $S_{2}$ surface dominate the dynamics.

This gives rise to the following question: Is there a way, by making use of design rules, to slow down the hot exciton deactivation processes, thus reducing the $H E \rightarrow L E$ relaxation and increasing the lifetime of high-energy states, which serve as a gateway to CT states?

\section{Design rules for long-lived hot $\mathrm{CT}$ states}

A possible answer to this question is suggested by additional TDDFT nonadiabatic dynamics simulations on the bi-thiophene dimer, in which the Cartesian coordinates of the sulphur atoms and the nearest bonded carbons are frozen, as indicated in Figure 6 (where only one bi-thiophene molecule is shown for clarity). In these constrained simulations (in which the $\mathrm{C}-\mathrm{H}$ bonds and the $\mathrm{C}-\mathrm{C}$ bonds in the 
thiophene ring are still free to oscillate), there are still ultrafast nonadiabatic transitions within the HE band, but those from the HE to the LE band are completely suppressed (Figure 6), as expected because of the artificial constraint on the CS distances. This supports our hypothesis that CS stretching vibrations are associated to the ultrafast relaxation within the manifold of excited states.

The exciton transfer from $\mathrm{HE}$ to $\mathrm{LE}$ is ruled by the energy gap $\Delta E\left(\mathrm{~S}_{3}{ }^{\mathrm{AB}}-\mathrm{S}_{2}{ }^{\mathrm{AB}}\right)$. In Figure 6 we compare the gaps obtained from the constrained dynamics (red line) and from all unconstrained trajectories reaching the maximum simulation time (grey lines). For the constrained dynamics, the computed gaps oscillate between 0.55 and $0.20 \mathrm{eV}$, while they vary over a much larger range in the unconstrained case, often reaching values as small as $0.01 \mathrm{eV}$. By constraining the CS bond lengths, the $\mathrm{S}_{3}{ }^{\mathrm{AB}} \rightarrow \mathrm{S}_{2}{ }^{\mathrm{AB}}$ crossing is evidently inhibited, with a concomitant increase in the lifetime of the highenergy excited states in the HE band.
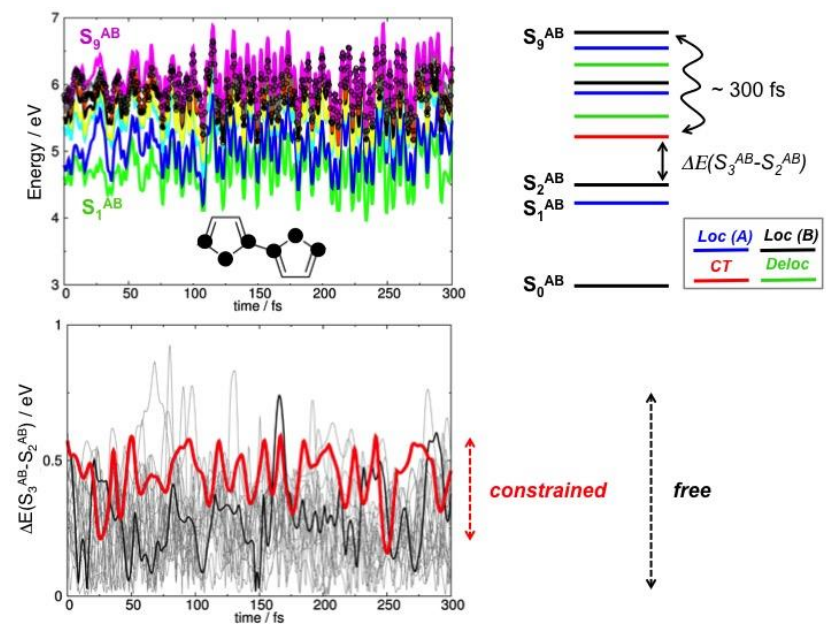

Figure 6. Top, left: TD- $\omega \mathrm{B} 97 \mathrm{XD} / 6-31 \mathrm{G}^{*}$ high-energy nonadiabatic excited state dynamics of a bi-thiophene dimer, for a trajectory with frozen Cartesian coordinates of the carbon and sulfur atoms (highlighted with black dots in the molecular structure). Top, right: sketch of the excited states involved in the deactivation process. Bottom: evolution of the gaps $\Delta E\left(\mathrm{~S}_{3}{ }^{\mathrm{AB}}-\mathrm{S}_{2}{ }^{\mathrm{AB}}\right)$ in all trajectories reaching the maximum simulation time in the unconstrained dynamics (grey lines), in the constrained trajectories with frozen coordinates (red line), and in trajectory A (black, see text).

The simple intuitive design rule emerging from these restricted-dynamics simulations is that the lifetime of hot excitons ${ }^{24,25}$ should be increased by finding ways to reduce the amplitude of the CS 
vibrations. Considering that the quantum oscillator amplitude is given by $(\hbar / \mu \omega)^{1 / 2}$, where $\mu$ is the reduced mass and $\omega$ the angular frequency, this could be achieved by increasing either $\mu$ or $\omega$, for example by introducing into the molecular backbone isoelectronic heavy atoms (e.g. Se vs. S, or Si vs. $\mathrm{C}$ as recently reported ${ }^{63}$, increase in $\mu$ ), or fused thiophene rings which flatten the structure and enhance the local C-S electron density (e.g. thienoacene units, increase in $\omega$ ).

We note that these proposed chemical modifications are motivated only by qualitative arguments and that we do not yet have any computational data to support them. Moreover, they focus exclusively on the control of the nuclear vibrations, without considering their impact on the electronic structure.

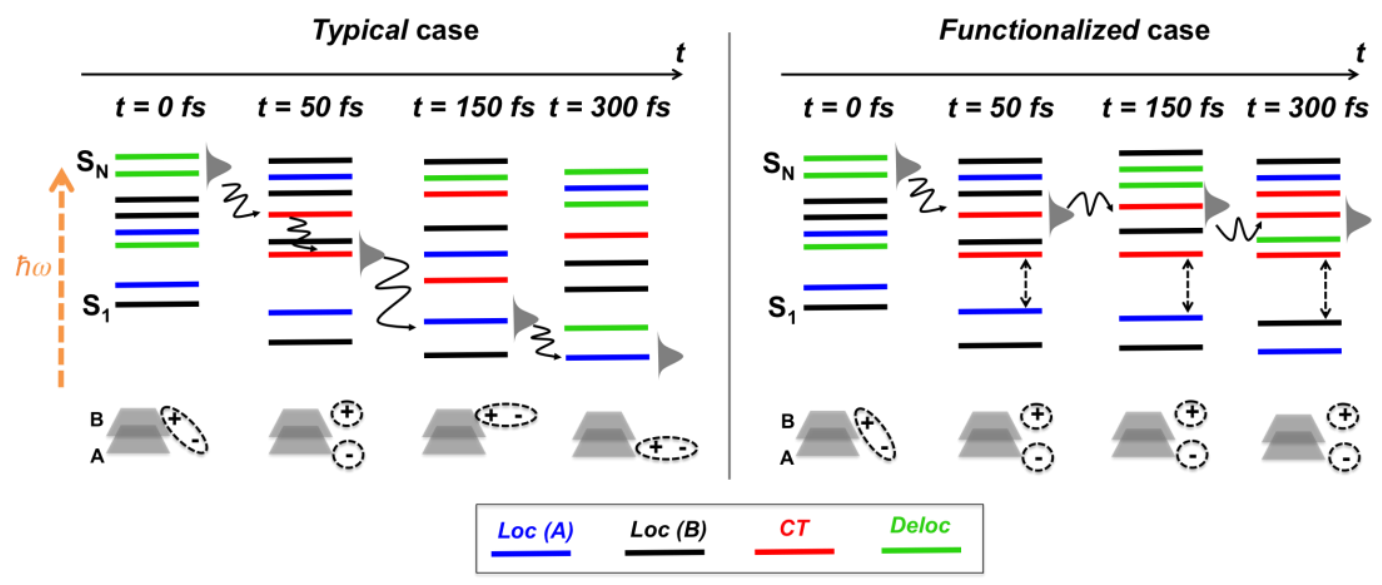

Figure 7. Schematic representation and classification of the excited states in an organic aggregate at different time steps during the excited-state dynamics. Left side: typical thiophene-based aggregate, with a sketch of the computed ultrafast deactivation via hot CT states. Right side: functionalized aggregate, with a sketch of the processes that tend to increase the lifetime of hot excitons (thus favouring CT processes) and to keep a high $(\geq 0.2 \mathrm{eV})$ and constant energy gap $\Delta E\left(\mathrm{~S}_{3}{ }^{\mathrm{AB}}-\mathrm{S}_{2}{ }^{\mathrm{AB}}\right)$ (thus disfavouring exciton transfer to the LE regime).

In Figure 7 (left side) we schematically sketch and summarize our findings. For (typical) thiophene-based aggregates, the initial high-energy exciton evolves via nonadiabatic transitions to hot CT states that are populated in tens of femtoseconds. Hot CT states last for $\sim 100 \mathrm{fs}$, and then decay to low-lying excited states. At the bottom of the excited-state LE band, the exciton oscillates between localized and delocalized states without ever returning to a CT state. For a hypothetical functionalized 
aggregate where the HE and LE bands are kept energetically apart (Figure 7 right side), the exciton is trapped in the HE band and does not populate the LE band (on an ultrafast time scale), which would increase the probability to populate CT states.

The readjustment of the electronic structure due to repopulation of excited states favoring specific relaxation channels resembles the so-called shishiodoshi transfer mechanism, which denotes a concerted unidirectional electronic and vibrational energy transfer through a chain of excited states with descending energy; this process is enhanced by vibronic relaxations ${ }^{7}$.

The ultrafast decay via hot CT states, observed in our full-dimensional TDDFT nonadiabatic dynamics, has important consequences for thiophene-based homo- and heterogeneous organic interfaces used for electronic and photovoltaic applications. The possibility to populate in few femtoseconds a high-energy CT state that may live over longer times opens the way for ultrafast electron transfer and charge separation, before relaxation to the bottom of the interfacial excited states can happen.

\section{CONCLUSION}

Full-dimensional nonadiabatic excited-state dynamics based on linear-response TDDFT allow us to gain insight on the photophysics of high-energy (hot) excitons at organic/organic interfaces. Ultrafast exciton deactivation processes were investigated for a bi-thiophene dimer, considered as representative of a large class of molecular aggregates, homogeneous and heterogeneous organic interfaces. The time scales and relaxation processes emerging from our TDDFT simulations nicely agree with data from state-of-the-art ultrafast spectroscopy on small thiophene-based conjugated molecules, acenes, homoand co-polymers, and even on polymer/fullerene interfaces $22,25,26,32,64-68$. Our findings are of general relevance for the interpretation and understanding of ultrafast excitonic processes in functional conjugated molecular aggregates, and for the design of new efficient solid-state photoactive materials.

In the early stages of the relaxation process ( 20-50 fs), ultrafast nonadiabatic transitions occur within the manifold of high-energy excited states, populating hot CT states. Hot CTs live for $\sim 100 \mathrm{fs}$ and then decay to low-energy states, namely $\mathrm{S}_{2}{ }^{\mathrm{AB}}$ and $\mathrm{S}_{1}{ }^{\mathrm{AB}}$. Thereafter, the system oscillates between 
localized and delocalized states, in a process governed by CS vibrations. Already at $300 \mathrm{fs}, 80 \%$ of the occupation is transferred to the bottom of the excited-state manifold, from where nonradiative decay processes through $S_{1} / S_{0}$ crossings occur, but much less frequently than in the monomer case ${ }^{56,59}$. This can be traced back to an energy barrier between the $S_{1}$ minimum and the $S_{1} / S_{0}$ crossing, which impedes non-radiative $\mathrm{S}_{1} / \mathrm{S}_{0}$ deactivation in the $\mathrm{vdW}$ dimer.

Nonadiabatic transitions within the manifold of the excited states are fostered by CS vibrations. CS bond elongations stabilize the excited states, thus promoting fast depopulation of hot CT states and reducing the energy gap between $\mathrm{HE}$ and LE bands (via pronounced stabilization of the $\mathrm{S}_{3}$ state).

Increasing the lifetime of hot excitons is a possible way to enhance the occupation of the CT states, which may lead to polaron formation in molecular aggregates. We explored this possibility by running constrained nonadiabatic excited-state dynamics, in which the promoting CS vibrations were suppressed by freezing the corresponding coordinates. This prevented the ultrafast population of lowlying excited states (Figure 6), without inhibiting nonadiabatic transitions within the high-energy excited-state manifold. The resulting increase in the lifetime of hot excitons improves the odds of populating long-lived hot CT states (Figure 7).

Based on these findings, we conclude that the search for ways to extend hot-exciton lifetimes may be a productive goal in the design of molecular aggregates for photovoltaics. For example, focusing on the mechanics of the nuclear frame, we anticipate that replacement of Se for S (or maybe Si for C) in the molecular backbone may mechanically reduce the amplitude of the relevant promoting vibrations and that introduction of fused thiophene rings (e.g. thienoacene units) ${ }^{69}$ may lead to a higher electron density in the CS bonds and strengthen them; these measures may increase the lifetime of hot excitons and open an energy gap between the HE and LE bands, thus impeding nonadiabatic HE-LE transitions (with localization and recombination). However, we note that these measures will also impact the electronic structure, in ways that we have not yet evaluated.

Independently of the success of such heuristic approaches, we emphasize that the bi-thiophene dimer investigated in this work should be understood as a prototype for photoinduced charge-separation in vdW organic dimers. Although it is gratifying that our simulations agree with the measured photo- 
induced charge transfer time constants in organic crystals and semiconducting polymers, we believe that the most interesting aspect of our study is the demonstration of how the first-principles characterization of the time evolution of the electronic structure may lead to new insights on molecular design. Rather than expecting larger complexes to share the same electronic features as our small prototype, we believe that the goal should be the following: given a certain organic crystal, we should identify and locate the CT states within the manifold of the electronically excited states relevant to ultrafast relaxation, and then try to devise ways to increase their lifetime by isolating them at the bottom of the electronic band. We believe that this approach is a true step forward in relation to the common analysis based on HOMO-LUMO gaps and vertical excitation spectra.

Future work will need to focus on the investigation of singlet-state dynamics in larger molecular aggregates and at donor/acceptor interfaces ${ }^{32}$ and on singlet-triplet exciton dynamics ${ }^{70}$. This will require nonadiabatic excited-state dynamics simulations with theoretical methods, such as linear response TDDFT, that are able to reasonably describe the excited-state electronic structure ${ }^{39,71}$ of medium-large systems.

\section{ASSOCIATED CONTENT}

Supporting Information. Supplementary Note 1: ground state optimized geometries, excited state (singlet and triplet) vertical transition energies, double excitations, and absorption spectra. Supplementary Note 2: details on the computational methods and fitting procedure for the HE and LE state population. Supplementary Note 3: time-dependent oscillations of internal coordinates (ring puckering, C-C bond length and bithiophene dihedral angle oscillations); electron densities evaluated along traj A, traj B and trajectory with frozen degrees of freedom; energy of the triplet states along the dynamics; and $S_{1}$ relaxed potential energy profiles for different values of the inter-molecular dimer distance. Supplementary Note 4: optimized Cartesian coordinates and absolute energies. 


\section{AUTHOR INFORMATION}

\section{Corresponding Authors}

fazzi@kofo.mpg.de; mario.barbatti@univ-amu.fr; thiel@kofo.mpg.de

\section{Author Contributions}

All authors contributed to analyse the data and write the manuscript.

\section{FUNDING SOURCES}

No competing financial interests have been declared.

\section{ACKNOWLEDGMENTS}

D.F. acknowledges the Alexander von Humboldt foundation for a postdoctoral research fellowship. M.B. thanks the support from the A*MIDEX grant ( ${ }^{\circ}$ ANR-11-IDEX-0001-02) and from the project Equip@Meso (ANR-10-EQPX-29-01), both funded by the French Government «Investissements d'Avenir» program.

\section{REFERENCES}

(1) Zewail, A. H. J. Phys. Chem. A 2000, 104, 5660.

(2) Long, R.; Prezhdo, O. V. Nano Lett. 2014, 14, 3335.

(3) Junhyeok Bang, S. M., Yi-Yang Sun, Damien West, Zhiguo Wang, Fei Gao, and S.

B. Zhang Proc. Natl. Acad. Sci. USA 2013, 110, 908.

(4) Oldani, N.; Tretiak, S.; Bazan, G.; Fernandez-Alberti, S. Energy \& Environmental Science 2014, 7, 1175.

(5) Gregory D. Scholes, G. R. Nat. Mater. 2006, 5, 14.

(6) Collini, E. Chem. Soc. Rev. 2013, 42, 4932.

(7) Nelson, T.; Fernandez-Alberti, S.; Roitberg, A. E.; Tretiak, S. Acc. Chem. Res. 2014, 47, 1155 
(8) Collini, E.; Scholes, G. D. Science 2009, 323, 369.

(9) Watanabe, S.; Ando, K.; Kang, K.; Mooser, S.; Vaynzof, Y.; Kurebayashi, H.; Saitoh, E.; Sirringhaus, H. Nat. Phys. 2014, 10, 308.

(10) Akimov, A. V.; Neukirch, A. J.; Prezhdo, O. V. Chem. Rev. 2013, 113, 4496.

(11) Clarke, T. M.; Durrant, J. R. Chem. Rev. 2010, 110, 6736.

(12) Jean-Luc Brédas, J. E. N., Jérôm Cornil, Veaceslav Coropceanu Acc. Chem. Res. 2009, 42, 1691.

(13) Clark, J.; Nelson, T.; Tretiak, S.; Cirmi, G.; Lanzani, G. Nat. Phys. 2012, 8, 225.

(14) Bubnova, O.; Khan, Z. U.; Wang, H.; Braun, S.; Evans, D. R.; Fabretto, M.; HojatiTalemi, P.; Dagnelund, D.; Arlin, J. B.; Geerts, Y. H.; Desbief, S.; Breiby, D. W.; Andreasen, J. W.; Lazzaroni, R.; Chen, W. M.; Zozoulenko, I.; Fahlman, M.; Murphy, P. J.; Berggren, M.; Crispin, X. Nat. Mater. 2014, 13, 190.

(15) Akselrod, G. M.; Deotare, P. B.; Thompson, N. J.; Lee, J.; Tisdale, W. A.; Baldo, M. A.; Menon, V. M.; Bulovic, V. Nat. Commun. 2014, 5, 3646.

(16) Noriega, R.; Rivnay, J.; Vandewal, K.; Koch, F. P.; Stingelin, N.; Smith, P.; Toney, M. F.; Salleo, A. Nat. Mater. 2013, 12, 1038.

(17) Song, Y.; Clafton, S. N.; Pensack, R. D.; Kee, T. W.; Scholes, G. D. Nat. Commun. 2014, 5, 4933.

(18) Provencher, F.; Berube, N.; Parker, A. W.; Greetham, G. M.; Towrie, M.; Hellmann, C.; Cote, M.; Stingelin, N.; Silva, C.; Hayes, S. C. Nat. Commun. 2014, 5, 4288.

(19) Vázquez, H.; Troisi, A. Phys. Rev. B 2013, 88.

(20) Bera, S.; Gheeraert, N.; Fratini, S.; Ciuchi, S.; Florens, S. Phys. Rev. B 2015, 91.

(21) Gelinas, S.; Rao, A.; Kumar, A.; Smith, S. L.; Chin, A. W.; Clark, J.; van der Poll, T. S.; Bazan, G. C.; Friend, R. H. Science 2014, 343, 512.

(22) Najafov, H.; Lyu, B.; Biaggio, I.; Podzorov, V. Phys. Rev. B 2008, 77.

(23) Jiamo Guo, H. O., Hiroaki Benten, and Shinzaburo Ito J. Am. Chem. Soc. 2009, 131, 16869. 
(24) Grancini, G.; Maiuri, M.; Fazzi, D.; Petrozza, A.; Egelhaaf, H. J.; Brida, D.; Cerullo, G.; Lanzani, G. Nat. Mater. 2013, 12, 29.

(25) Bakulin, A. A.; Rao, A.; Pavelyev, V. G.; van Loosdrecht, P. H.; Pshenichnikov, M. S.; Niedzialek, D.; Cornil, J.; Beljonne, D.; Friend, R. H. Science 2012, 335, 1340.

(26) Paraecattil, A. A.; Banerji, N. J. Am. Chem. Soc. 2014, 136, 1472.

(27) Banerji, N. J. Mat. Chem. C 2013, 1, 3052.

(28) Moses, D. Nat. Mater. 2014, 13, 4.

(29) Tavernelli, I. Acc. Chem. Res. 2015, 48, 792.

(30) Wang, L.; Long, R.; Prezhdo, O. V. Ann. Rev. Phys. Chem. 2015, 66, 549.

(31) Rossky, F. S. a. P. J. J. Phys. Chem. B 2008, 112, 11.

(32) Jailaubekov, A. E.; Willard, A. P.; Tritsch, J. R.; Chan, W. L.; Sai, N.; Gearba, R.; Kaake, L. G.; Williams, K. J.; Leung, K.; Rossky, P. J.; Zhu, X. Y. Nat. Mater. 2013, 12, 66.

(33) Tamura, H.; Bittner, E. R.; Burghardt, I. J. Chem. Phys. 2007, 126, 021103.

(34) Tamura, H.; Burghardt, I. J. Am. Chem. Soc. 2013, 135, 16364.

(35) Nelson, T.; Fernandez-Alberti, S.; Chernyak, V.; Roitberg, A. E.; Tretiak, S. J. Phys. Chem. B 2011, 115, 5402.

(36) Wang, L.; Prezhdo, O. V.; Beljonne, D. Phys. Chem. Chem. Phys. 2015, 17, 12395.

(37) Liu, J.; Neukirch, A. J.; Prezhdo, O. V. J. Phys. Chem. C 2014, 118, 20702.

(38) Tapavicza, E.; Bellchambers, G. D.; Vincent, J. C.; Furche, F. Phys. Chem. Chem. Phys. 2013, 15, 18336.

(39) Sen, K.; Crespo-Otero, R.; Weingart, O.; Thiel, W.; Barbatti, M. J. Chem. Theory Comput. 2013, 9, 533.

(40) Caruso, D.; Troisi, A. Proc. Natl. Acad. Sci. USA 2012, 109, 13498.

(41) Dreuw, A.; Wormit, M. Wiley Interdisciplinary Reviews: Computational Molecular Science 2015, 5, 82.

(42) Barbatti, M.; Ruckenbauer, M.; Plasser, F.; Pittner, J.; Granucci, G.; Persico, M.; Lischka, H. Wiley Interdisciplinary Reviews: Computational Molecular Science 2014, 4, 26.

(43) Granucci, G.; Persico, M. J. Chem. Phys. 2007, 126, 134114. 
(44) Fernandez-Alberti, S.; Roitberg, A. E.; Nelson, T.; Tretiak, S. J. Chem. Phys. 2012, $137,014512$.

(45) Wang, L.; Prezhdo, O. V. J Phys. Chem. Lett. 2014, 5, 713.

(46) Granucci, G.; Persico, M.; Toniolo, A. J. Chem. Phys. 2001, 114, 10608.

(47) Plasser, F.; Granucci, G.; Pittner, J.; Barbatti, M.; Persico, M.; Lischka, H. J. Chem. Phys. 2012, 137, 22A514.

(48) Wang, L.; Beljonne, D. J. Phys. Chem. Lett. 2013, 4, 1888.

(49) Wang, L.; Trivedi, D.; Prezhdo, O. V. J. Chem. Theory Comput. 2014, 10, 3598.

(50) Hammes-Schiffer, S.; Tully, J. C. J. Chem. Phys. 1994, 101, 4657.

(51) Casida, M. In Recent advances in density functional methods, Part I; Chong, D., Ed.; World Scientific: Singapore, 1995, p 155.

(52) Barbatti, M.; Pittner, J.; Pederzoli, M.; Werner, U.; Mitrić, R.; Bonačić-Koutecký, V.; Lischka, H. Chem. Phys. 2010, 375, 26.

(53) Mališ, M.; Loquais, Y.; Gloaguen, E.; Biswal, H. S.; Piuzzi, F.; Tardivel, B.; Brenner, V.; Broquier, M.; Jouvet, C.; Mons, M.; Došlić, N.; Ljubić, I. J. Am. Chem. Soc. 2012, 134, 20340.

(54) Werner, U.; Mitrić, R.; Suzuki, T.; Bonačić-Koutecký, V. Chem. Phys. 2008, 349, 319.

(55) Tapavicza, E.; Tavernelli, I.; Rothlisberger, U. Phys. Rev. Lett. 2007, 98, 023001.

(56) Fazzi, D.; Barbatti, M.; Thiel, W. Phys. Chem. Chem. Phys. 2015, 17, 7787.

(57) Crespo-Otero, R.; Barbatti, M. Theo. Chem. Acc. 2012, 131, 1237.

(58) Schnell, M.; Erlekam, U.; Bunker, P. R.; von Helden, G.; Grabow, J.-U.; Meijer, G.; van der Avoird, A. Angewandte Chemie International Edition 2013, 52, 5180.

(59) Prlj, A.; Curchod, B. F.; Corminboeuf, C. Phys. Chem. Chem. Phys. 2015, 17, 14719.

(60) Weinkauf, R.; Lehr, L.; Schlag, E. W.; Salzmann, S.; Marian, C. M. Phys. Chem. Chem. Phys. 2008, 10, 393.

(61) Settels, V.; Schubert, A.; Tafipolski, M.; Liu, W.; Stehr, V.; Topczak, A. K.; Pflaum, J.; Deibel, C.; Fink, R. F.; Engel, V.; Engels, B. J. Am. Chem. Soc. 2014, 136, 9327. 
(62) Milani, A.; Brambilla, L; Del Zoppo, M.; Zerbi, G. J. Phys. Chem. B 2007, 111, 1271.

(63) Li, L.; Matsuo, T.; Hashizume, D.; Fueno, H.; Tanaka, K.; Tamao, K. J. Am. Chem. Soc. 2015.

(64) Fazzi, D.; Grancini, G.; Maiuri, M.; Brida, D.; Cerullo, G.; Lanzani, G. Phys. Chem. Chem. Phys. 2012, 14, 6367.

(65) Hwang, I.; Beaupré, S.; Leclerc, M.; Scholes, G. D. Chem. Sci. 2012, 3, 2270.

(66) Vandewal, K.; Albrecht, S.; Hoke, E. T.; Graham, K. R.; Widmer, J.; Douglas, J. D.; Schubert, M.; Mateker, W. R.; Bloking, J. T.; Burkhard, G. F.; Sellinger, A.; Frechet, J. M.; Amassian, A.; Riede, M. K.; McGehee, M. D.; Neher, D.; Salleo, A. Nat. Mater. 2014, 13, 63.

(67) Banerji, N.; Gagnon, E.; Morgantini, P.-Y.; Valouch, S.; Mohebbi, A. R.; Seo, J.-H.; Leclerc, M.; Heeger, A. J. J. Phys. Chem. C 2012, 116, 11456.

(68) Scarongella, M.; Laktionov, A.; Rothlisberger, U.; Banerji, N. J. Mat. Chem. C 2013, $1,2308$.

(69) Cinar, M. E.; Ozturk, T. Chem. Rev. 2015, 115, 3036.

(70) Cui, G.; Thiel, W. J. Chem. Phys. 2014, 141, 124101.

(71) Laurent, A. D.; Jacquemin, D. Int. J. Quant. Chem. 2013, 113, 2019. 